\title{
Modeling and simulation of a wirelessly-powered thermopneumatic micropump for drug delivery applications
}

\author{
Marwan Nafea ${ }^{1}$, Jeevananthan Baliah ${ }^{2}$, Mohamed Sultan Mohamed Ali ${ }^{3}$ \\ ${ }^{1}$ Department of Electrical and Electronic Engineering, University of Nottingham Malaysia, Malaysia \\ ${ }^{2}$ Huangpu Economic and Technological Development District, China \\ ${ }^{3}$ School of Electrical Engineering, Universiti Teknologi Malaysia, Malaysia
}

\begin{tabular}{l} 
Article Info \\
\hline Article historys: \\
Received Jan 27, 2019 \\
Revised May 5, 2019 \\
Accepted May 16, 2019 \\
\hline
\end{tabular}

\section{Keywords:}

Drug delivery

Microfluidic

Micropump

Thermopneumatic

Wireless power transfer

\begin{abstract}
This paper presents modeling and finite element analysis of a thermopneumatic micropump with a novel design that does not affect the temperature of the working fluid. The micropump is operated by activating a passive wireless heater using wireless power transfer when the magnetic field is tuned to match the resonant frequency of the heater. The heater is responsible for heating an air-heating chamber that is connected to a loading reservoir through a microdiffuser element. The solution inside the reservoir is pumped through a microchannel that ends with an outlet hole. The thermal and pumping performances of the micropump are analyzed using finite element method over a low range of Reynold's number $\leqslant 10$ that is suitable for various biomedical applications. The results demonstrate promising performance with a maximum flow rate of $\sim 2.86 \mu \mathrm{L} / \mathrm{min}$ at a chamber temperature of $42.5^{\circ} \mathrm{C}$, and a maximum pumping pressure of $406.5 \mathrm{~Pa}$. The results show that the developed device can be potentially implemented in various biomedical areas, such as implantable drug delivery applications.
\end{abstract}

Copyright (C) 2019 Institute of Advanced Engineering and Science. All rights reserved.

\section{Corresponding Author:}

Mohamed Sultan Mohamed Ali, School of Electrical Engineering, Faculty of Engineering, Universiti Teknologi Malaysia, 81310 Skudai, Johor, Malaysia. Email: sultan_ali@fke.utm.my

\section{INTRODUCTION}

Since the beginning of MEMS-development, micropumps were among the first devices to be fabricated in microscale, due to their various applications in microelectronics cooling, medicine, biology, and space exploration [1]. In various MEMS-based systems, the ability to precisely manipulate and pump small volumes of fluids is crucial. For instance, in biological systems, samples are required to be pumped through the components of miniature assay systems, which therefore requires achieving precise control of the pumping and manipulation process [2]. Another important aspect for microfluidic systems is the ability to possess a miniature self-contained micropump. For example, transdermal drug delivery systems require having small sizes that allow portable and implantable applications while achieving precise pumping of drugs compounds through the skin to reach the systemic circulation for subsequent distribution in the human body [3]. Several types of MEMS-based micropumps have been reported in the literature to address the aforementioned issues. Generally, micropumps are classified based on their pumping principle into two main categories. The first category is called mechanical or displacement micropumps, which have moving mechanical parts to exert pressure on the working fluid. The second category is called non-mechanical or dynamic micropumps, which 
add momentum to the working fluid, where this momentum is subsequently converted into pressure. These two categories of micropumps are subdivided into more categories, based on their actuation and construction methods [1]. However, the majority of reported micropumps are mechanical, since the performance of nonmechanical micropumps is highly dependent on properties of the working liquid, such as the conductivity of the fluid [4]. The most common type of mechanical micropumps is the diaphragm single chamber type, with a diffuser-nozzle (valveless) geometry. However, this type of micropumps has limitations in terms of the applications that are sensitive to vibrations or those requiring a continuous single-stroke delivery [2].

To address these issues, aperiodic (single-stroke) type micropumps are utilized. This type pumps a liquid from a pre-filled storage chamber into a microchannel, using the pressure generated by an actuator. Such aperiodic micropumps can be used to achieve a high-precision pumping without requiring moving parts, which makes them suitable for portable [5, 6] and disposable devices for biomedical applications [7, 8]. Pneumatic and thermopneumatic micropumps have been efficiently used in periodic and aperiodic applications [4]. Pneumatic micropumps require and external pressure source to perform the actuation process, while thermopneumatic micropumps utilize on-board heaters to actuate. This allows thermopneumatic micropumps to have a smaller sizes and simpler fabrication methods when compared to pneumatic micropumps. In addition, thermopneumatic microactuators offer an advantageous performance in terms of the combined efficiency and energy density when compared to other types of microactuators, such as piezoelectric and shape memoty alloy microactuators [9-11]. Thermopneumatic micropumps are be operated using the deflection of a membrane that is generated by heating and cooling the air trapped within a heating chamber [12]. The flow direction is usually controlled using active valves, passive check valves, or multiple actuators that generate peristaltic pumping. Nevertheless, such methods tend to limit the longevity of the micropump due to the tearing and aging effects of the moving elements. In addition, they are prone to bubbles trapping within the moving boundaries [1]. Single stroke micropumps can potentially address these issues by pushing the liquid from the reservoir to the outlet depending on the volume expansion of the air trapped within the heating chamber, which eliminates the risks of mechanical failures of the moving elements [7]. However, thermopneumatic micropumps require maintaining the operating power above a certain level to maintain pumping, which leads to a high-power consumption. This issue has been addressed by several researchers using efficient structures and signal with optimal frequencies. Yang and Lin [13] presented a valveless thermopneumatic micropump that operates using a vapor from a liquid that is stored in an evaporation chamber. Flow rate of $12.5 \mathrm{~nL} / \mathrm{min}$ was achieved using 4 VPP heating signal, which generated temperatures below $50{ }^{\circ} \mathrm{C}$. Yoo et al. [14] integrated thermopneumatic micropump with paraffin-actuated microvalves on the same substrate to achieve a maximum flow rate of 2.0 $\mu \mathrm{l} / \mathrm{min}$ using a $1 \mathrm{~Hz}$ signal with a power of $500 \mathrm{~mW}$. Chia et al. [12] presented a thermopneumatic peristaltic micropump with 3 chambers. A maximum flow rate of $20.01 \mu \mathrm{L} / \mathrm{min}$ was achieved using a $1.2 \mathrm{~Hz}$ with an amplitude of $9 \mathrm{~V}$ that generated a temperature below $91.9^{\circ} \mathrm{C}$.

However, the applications of thermopneumatic micropumps in implantable medical devices are limited, due to the limitation of mobility of these devices when using wires and conventional powering methods. To date, the alternative solutions of this problem are using on-board batteries, magnets, biofuel cells, or nuclear cells, which increase the size of these devices and limit their application range and operation time [15]. Passively driven actuators can be used to solve the aforementioned drawbacks while offering a higher biocompatibility and robustness, smaller size, and lower cost compared to actively driven actuators [16, 17]. Wireless actuators have been utilized in several passive microfluidic applications, such as implantable drug delivery devices, and a microsyringe that is controlled by multiple shape-memory-alloy actuators [18]. This paper presents an extended study of our previously reported wirelessly controlled passive thermopneumatic micropump [19, 20]. Dynamic heat transfer, thermopneumatic actuation and pumping performances are analyzed using finite element method.

\section{DESIGN AND WORKING PRINCIPLE}

The device comprises a planar inductor-capacitor (LC) wireless heater, with a spiral inductor of 5 $\mathrm{mm} \times 5 \mathrm{~mm}$, and a capacitor of $5 \mathrm{~mm} \times 2.5 \mathrm{~mm}$. The heater is attached beneath an air-heating chamber with similar dimensions to the wireless heater. The air chamber is connected to a $5 \mathrm{~mm}$-diameter loading reservoir using a $10^{\circ}$ microdiffuser that rectifies the flow of the fluid. The reservoir is connected to a microchannel with a length of $4 \mathrm{~mm}$ that ends an outlet hole with a diameter of $2.5 \mathrm{~mm}$. The device pumps the liquid in a single direction without requiring any mechanical moving membrane, which eliminates the leakage flow of the diffuser. The microchannel layer is covered with a $2 \mathrm{~mm}$-thick cover lid to decrease the deflection and possible air outflow from the air heating chamber to the surrounding. Figure 1 illustrates the construction design of the developed micropump.

Pumping in the developed micropump is accomplished through the heat transferred from the planar LC wireless heater (Figure 1, which serves as a frequency-dependent actuator). The passive resonant circuit is 
formed by connecting the parallel plate capacitor and the planar inductor in parallel, where the inductor has a width of $w_{C}$, a separation gap of $s_{C}$, and a parasitic capacitance of $C_{p a r}$, as illustrated in Figure 2.

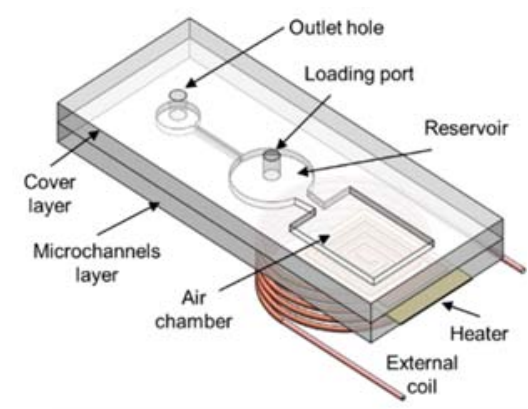

Figure 1. Schematic diagram of the thermopneumatic micropump

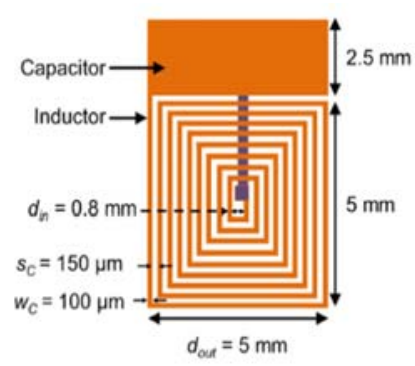

(a)

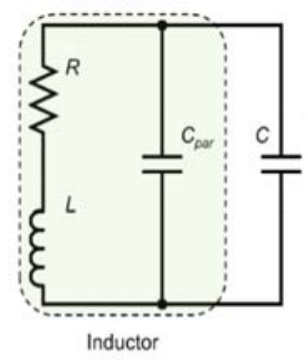

(b)

Figure 2. (a) Sample layout of the LC circuit design (not to scale) (b) equivalent circuit of the LC heater

When the magnetic field with a frequency $\left(f_{m}\right)$ is tweaked to the resonant frequency (fr) of the LC circuit, the resonant circuit can be magnetically coupled to an external transmitting circuit. $f_{r}$ can be calculated from the formula below [21]:

$$
f_{r}=\frac{1}{2 \pi \sqrt{L C}}
$$

The values of $L$ and $C$ depend on their shape, dimensions, and material. A simplified accurate model for the planar spiral inductor based on the Wheeler model has been reported in [22] to facilitate the design of planar coils. The $L$ value of the square spiral coil as illustrated in Figure 2 is given by:

$$
L=\frac{K_{1} \mu_{0} n^{2} d_{\text {avg }}}{1+K_{2} \rho_{f r}}
$$

where the layout dependent constants $K_{1}$ and $K_{2}$ are 2.34 and 2.75, respectively. The magnetivity of free space $\left(\mu_{0}\right)$ is given as $0.4 \pi \mu \mathrm{H} / \mathrm{m}$, and $n$ is the number of turns. The fill ratio $\left(\rho_{f r}\right)$ is defined as $\left(d_{\text {out }}-d_{\text {in }}\right) /\left(d_{\text {out }}+d_{\text {in }}\right)$, and the average diameter $d_{\text {avg }}$ is given by $\left(d_{\text {out }}+d_{\text {in }}\right) / 2$, where $d_{\text {in }}$ and $d_{\text {out }}$ are as define in Figure 2 . Therefore, the resistance $(R)$ of the coil can be calculated as follows [23]:

$$
R=\frac{\rho l}{A_{C o}}
$$

where $\rho$ is the resistivity of the material, and $l$ and $A_{C o}$ are the length and cross-sectional area of the coil. The value of $C$ is determined by the area of the parallel plates $\left(A_{P l}\right)$, the separation gap $\left(d_{C}\right)$, and the dielectric constant of the dielectric material $\left(\varepsilon_{r}\right)$ between the plates of the capacitor. In addition, the parasitic capacitance of the coil is taken into account when calculating the total capacitance. This capacitance can be determined using the following formula [24]:

$$
C=\varepsilon_{0}\left[\frac{\varepsilon_{r} A_{P l}}{d_{C}}+\left(0.9+0.1 \times \varepsilon_{r}\right) \frac{d_{C}}{s_{C}}\left[4\left(d_{\text {out }}-w_{C} n\right)(n-1)-4 s_{C} n(n+1)\right]\right]
$$

The first part of (4) represents the capacitance of the parallel plates, and the second part represents the parasitic capacitance of the coil, where $\varepsilon_{0}$ is the vacuum permittivity. In this work, a single-sided copper-clad polyimide film with $d_{C}$ of $50 \mu \mathrm{m}$ and $\varepsilon_{r}$ of 3.4 was used to as a material for the LC circuit. Thus, from Figure 2 and $(1-4)$, it can be determined that the 9-turns planar coil has a theoretical $L$ of $\sim 231 \mathrm{nH}$ and $R$ of $\sim 3 \Omega$, while the value of $C$ is $7.81 \mathrm{pF}$ and $f_{r}$ of the circuit is $\sim 118.53 \mathrm{MHz}$.

When exposed to an external radio frequency magnetic field (generated from the external coil) that matches its $f_{r}$ [26], the LC wireless heater generates Joule heat. The power, $P$, consumed in the circuit of the LC can be expressed by the following equation [25]: 


$$
P(\omega)=\frac{R v^{2}}{[R+j(\omega L-1 / \omega C)]^{2}}
$$

where $\omega$ is the angular frequency of the AC current and $v$ is the electromotive force. When the field frequency is tuned to the resonant frequency of the circuit, the power transferred to the circuit is maximized. The reactance is eliminated under this condition, so that the maximum power transferred to the LC circuit is expressed as:

$$
P\left(f_{r}\right)=\frac{v^{2}}{R}
$$

Therefore, in the LC circuit, the produced Joule heat reaches the maximum value at $f_{r}$ of the LC circuit while maintaining the magnetic field intensity. The steady state temperature, $T_{S S}$, can be expressed as [26]:

$$
T_{S S}=\frac{R_{T} v^{2} / R}{1+\alpha_{R} R_{T} v^{2} / R}
$$

where $\alpha_{R}$ is the temperature coefficient of the resistance of the circuit, and $R_{T}$ is the thermal resistance to the surroundings, respectively. The generated heat is transferred through the PDMS layer below the chamber from the LC circuit to the air-heating chamber. The rate of change in temperature is related to the conduction of energy, $Q$ via the PDMS layer. This can be expressed by Fourier's equation, as follows:

$$
Q=\frac{k A_{P D M S} \Delta T}{\Delta x y}
$$

where $A_{P D M S}$ is the surface contact area between the LC heater and the corresponding air-heating chamber, $k$ is the thermal conductivity of the PDMS, and $\Delta x y$ and $\Delta T$ represent the thickness of the PDMS layer and the temperature variance across it, respectively. From (8) states clearly that by decreasing the thickness of the PDMS layer, the rate of conductive heat transfer can be maximized. A thinner layer, however, is more susceptible to deformation, reducing the pressure inside the air chamber. The LC heater is about three orders of magnitude higher than PDMS in this research [27], which makes it stiff and significantly decreases the bending of the bottom walls of the air chamber. When the heat is transferred to its walls, the air temperature inside the chamber increases, causing the volume of air to increase on the basis of Charles' law. This law states that when the pressure (p) and number of moles are kept constant, the volume $(V)$ of an ideal air is directly proportional to its absolute temperature. When the temperature increases to $T_{1}$, the volume of air $\left(V_{0}\right)$ in the chamber at room temperature $\left(T_{0}\right)$ increases to volume $\left(V_{1}\right)$ based to the following equation [28]:

$$
\Delta V=\frac{V_{0}}{T_{0}} \Delta T
$$

where $\Delta V=V_{1}-V_{0}$ and $\Delta T=T_{1}-T_{0}$. This formula can be further expressed by the ideal gas equation that describes the relationship between $p, V$, and the absolute temperature, $T$, of the gas:

$$
p V=N k_{B} T
$$

The number of air molecules in the air chamber is represented by $N$, while $k_{B}$ is the Boltzmann constant. Since there is a constant number of air molecules inside the air chamber, then it is possible to use (9) to express the relationship between $\Delta T$ and $\Delta V$. The change in air volume can therefore be determined by the chamber temperature elevation. Before the heating operation, the liquid loading hole was sealed with silicone adhesive to prevent the liquid from leaking through the hole. It should be noted that in the pumping process, the thermal power supplied to the air chamber, $\dot{E}$, is not fully used. The conservation of energy during the device operation requires that:

$$
\dot{E}=h_{a i r} \Delta T+C_{t h} \frac{d}{d t}(\Delta T)+\left(p_{0}+\Delta p\right) \Delta V
$$

where $C_{t h}, h_{\text {air }}$, and $\Delta p$ are the effective heat capacity of the air chamber, the effective heat transfer coefficient of the walls of the air-heating chamber to the surroundings, and the change in pressure inside the air-heating chamber, respectively [29]. The power transferred to the air-heating chamber is divided into three parts, as shown in (11). The first part represents the dissipated heat to the surroundings, the second part represents the heat stored in the air-heating chamber, and the last part represents the heat that is transformed into a mechanical power. This power causes a deformation in the walls of the air-heating chamber with an amplitude, $s$, which can be presented as [30]: 
Due to the thickness of the side walls of the air-heating chamber, only the top and bottom walls are subjected to deformation. In addition, due to of its rigid material in comparison with PDMS, the attached LC heater significantly reduces the deformation. The deformation can thus be approximated as of the top wall of the chamber:

$$
S \approx \frac{3 \Delta V}{A_{W}} \approx \frac{3 V_{0} \Delta T}{A_{W} T_{0}}
$$

where $A_{W}$ is the area of the top wall of the chamber. The deformation is greatly decreased in this work by increasing the thickness of the top wall of the air-heating chamber to $2 \mathrm{~mm}$. Thus, most of the mechanical boundary power part in (7) is applied to the inlet of the microdiffuser element, which significantly reduces the loss in thermal power transferred to the air-heating chamber.

\section{FINITE ELEMENT ANALYSIS}

In this work, COMSOL Multiphysics ${ }^{\circledR}$ was used to perform the numerical simulation of a threedimensional (3D) model of the developed device. In the simulation process, heat transfer, solid mechanics, and laminar flow physics were used. It was assumed that the initial air temperature was $23{ }^{\circ} \mathrm{C}$ without thermal losses to the environment. The heater temperature was selected on the basis of previously carried out experimental results in [31]. To guarantee the independency of the results of the analysis, a mesh sensitivity test with dfferent mesh densities was carried out. An advanced GMRES with multi-level multi-discretization solver was used to minimize the numerical error that arises from the discretization of the Navier-Stokes equation, which was solved based on the following equation $[32,33]$ :

$$
\rho\left[\frac{\partial u}{\partial t}+(u . \nabla) u\right]=-\nabla p+\mu \nabla^{2} u
$$

where $u$ and $\rho$ are the fluid velocity and density, respectively. The fluid dynamic viscosity and density were set to $10^{-6} \mathrm{~m}^{2} / \mathrm{s}$ and $999.8 \mathrm{~kg} / \mathrm{m}^{3}$, respectively. Thus, $q$ can be expressed as [34]:

$$
q=\frac{\Delta p A_{C} r^{2}}{8 \mu_{f} l_{C}}
$$

where $r$ is the diagonal length of the microchannel, $A_{C}$ is the cross-sectional area of the microchannel, and $l_{C}$ is the length of the microchannel. The fluid dynamics in the microscale are characterized by a dimensionless parameter, Reynolds number $(R e)$, which is defined as [35]:

$$
R e=\frac{\rho u D_{h}}{\mu}
$$

where $D_{h}$ is the hydraulic diameter of the microchannel. The simulation results are further presented and discussed in the following section.

\section{RESULTS AND DISCUSSION}

This section presents the numerical simulation results of the thermal behavior of the wireless thermopneumatic micropump. COMSOL Multiphysics ${ }^{\circledR}$ is used to perform finite element analysis of the heat distribution profile across the thermopneumatic micropump. The heater was activated with a power of $60 \mathrm{~mW}$ for $50 \mathrm{~s}$ until its temperature reached $50{ }^{\circ} \mathrm{C}$ and then deactivated for $190 \mathrm{~s}$ until its temperature reached room temperature $\left(23{ }^{\circ} \mathrm{C}\right)$ to investigate the heat transfer and thermal expansion behaviors.

Figure 3 shows the temperature distribution profile of the 3D dimensional model of the micropump after activating the heater for $50 \mathrm{~s}$. It can be noted from the figure that the fluid temperature inside the reservoir and microchannels is not affected by the heater temperature. This is an important aspect in biomedical devices that handle fluids that are sensitive to temperature changes. Furthermore, the thermal conduction behavior of the heater and the PDMS bottom wall of the air-heating chamber is investigated, as illustrated in Figure 4.

Indo. J. Elec. Eng. \& Inf, Vol.7, No. 2, June 2019: 182 - 189 


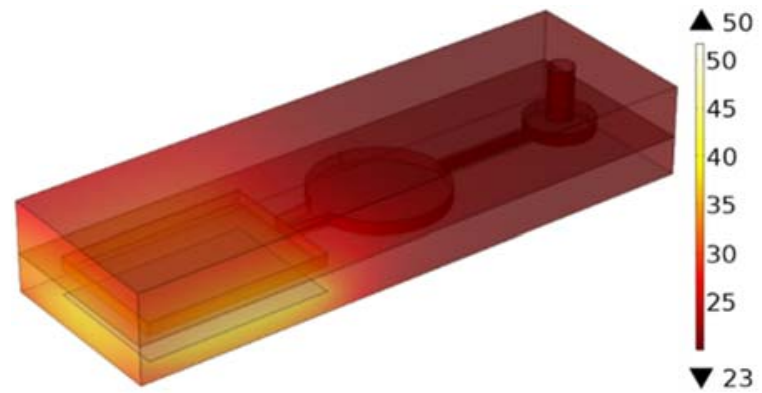

(a)

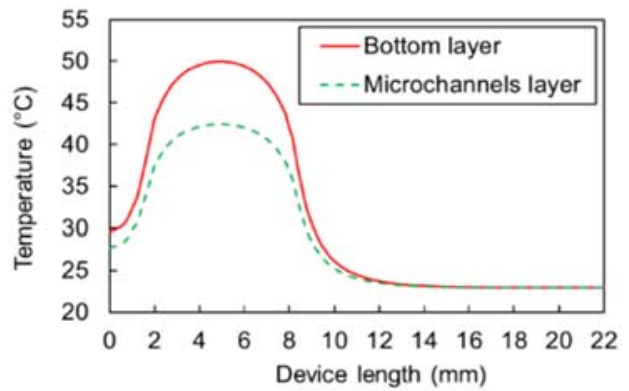

(b)

Figure 3. Simulation results. (a) 3D model of the micropump showing the temperature distribution profile of the device. (b) Temperature distribution profile along the bottom and microchannels layers of the device

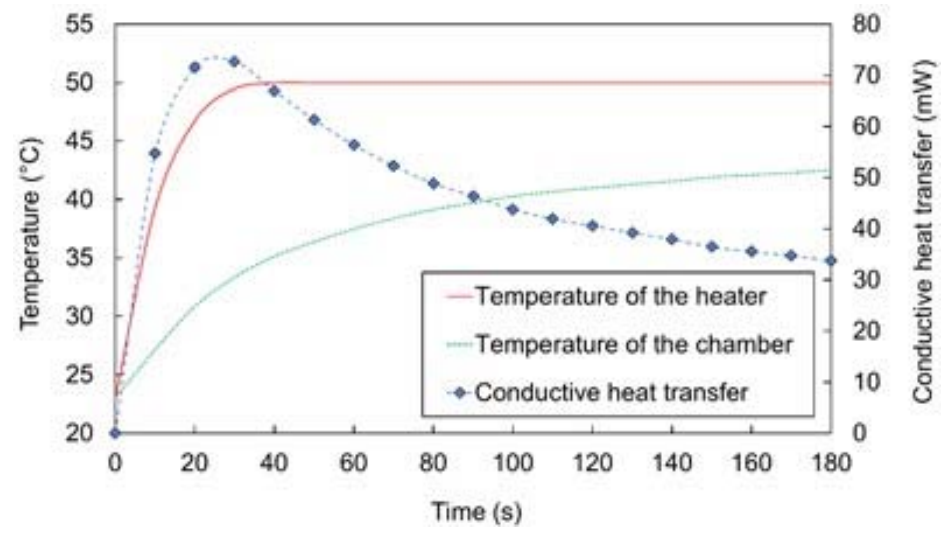

Figure 4. Dynamic heat transfer of the LC heater and the chamber

It can be seen that the heater temperature is increased to a steady state value of $\sim 50{ }^{\circ} \mathrm{C}$ in $\sim 40 \mathrm{~s}$, while the air chamber temperature required $170 \mathrm{~s}$ to reach a steady state of $42.5^{\circ} \mathrm{C}$. The conductive heat transfer, calculated from (8), reached a maximum value of $72.67 \mathrm{~mW}$ after approximately $30 \mathrm{~s}$. The rate of conductive heat transfer gradually decreased beyond that point until it reached $\sim 33.75 \mathrm{~mW}$. Due to the temperature generated in the heater, which is transferred to the bottom wall of the air chamber, the air temperature inside the air chamber is increased.

Figure 5(a) clearly demonstrates the aforementioned behavior, which shows that the air chamber temperature is directly affected by the heater temperature. The air chamber temperature reached a maximum value of $42.5{ }^{\circ} \mathrm{C}$ after activating the heater for $50 \mathrm{~s}$. Therefore, as the air temperature increased, a maximum deformation of $\sim 50 \mu \mathrm{m}$ was generated on the top surface of the air chamber. After deactivating the heater, the deformation gradually decreased as the air chamber temperature was equal to the room temperature until it reached its initial condition. In addition, 2 pumping strokes were used to investigate the pumping performance of the micropump, as shown in Figure 5(b). From the figure, to achieve each pumping stroke, it can be seen that the air chamber temperature was controlled by activating and deactivating the heater for $50 \mathrm{~s}$ each time. After 50 and $150 \mathrm{~s}$, a maximum flow rate of $2.86 \mu \mathrm{L} / \mathrm{min}$ was observed at each point when the air chamber temperature in both cases was at its maximum $42.5^{\circ} \mathrm{C}$. Furthermore, the figure clearly shows that the flow rate is directly affected by the air-heating chamber temperature and consequently by the wireless LC heater temperature. In addition, it is worth mentioning that the micropump achieved a maximum pumping pressure of $406.5 \mathrm{~Pa}$, demonstrating an appropriate performance for different biomedical applications. 


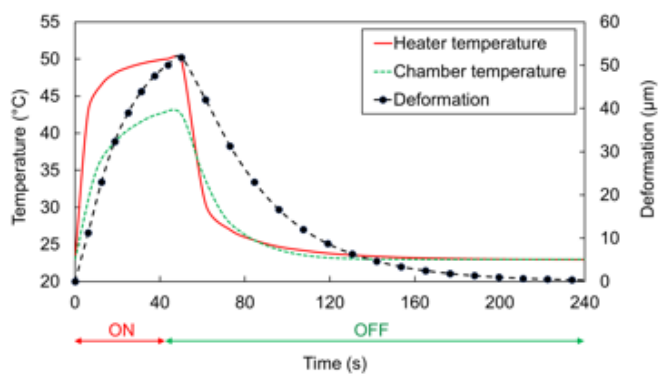

(a)

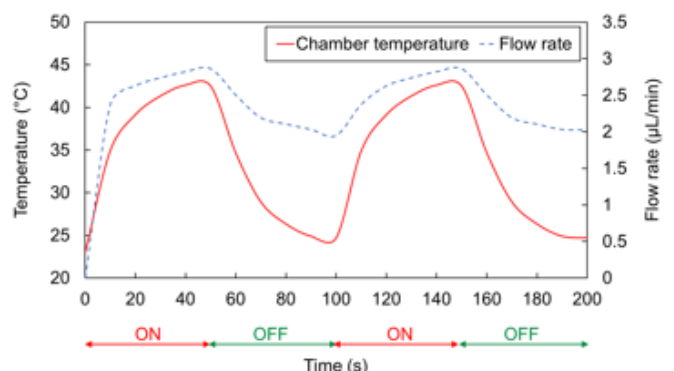

(b)

Figure 5. Temporal response of the (a) temperature of the heater and air chamber, and resultant deformation. (b) Pumping performance using 2 strokes

\section{CONCLUSION}

This paper presented a thermopneumatic micropump modeling and finite element analysis. Using an external magnetic field, the micropump was operated wirelessly to activate a passive wireless LC heater responsible for heating an air heating chamber. The performance of the micropump was investigated using a finite-element method over a low range of $\mathrm{Re} \leqslant 10$, which is suitable for biomedical applications. The operation of the device was investigated using single and double strokes, showing promising performance results with a maximum pumping pressure and flow rate of $406.5 \mathrm{~Pa}$ and $2.86 \mu \mathrm{L} / \mathrm{min}$ and respectively. The results showed that the micropump could potentially be implemented in different biomedical applications that require maintaining a stable temperature of the pumped solutions.

\section{ACKNOWLEDGEMENTS}

This work was supported by Research University Grant (GUP 17H61) from Universiti Teknologi Malaysia; and Prototype Development Research Grant Scheme (PRGS 4L690) from Ministry of Higher Education Malaysia.

\section{REFERENCES}

[1] D. Laser and J. Santiago, "A Review of Micropumps," Journal of Micromechanics and Microengineering, vol. 14, pp. R35-R64, 2004.

[2] M. N. Minjal, "Frequency-Controlled Wireless Passive Microfluidic Devices," Doctor of Philosophy, Faculty of Electrical Engineering, Universiti Teknologi Malaysia, Johor, Malaysia, 2018.

[3] M. W. Ashraf, S. Tayyaba, A. Nisar, N. Afzulpurkar, D. W. Bodhale, T. Lomas, et al., "Design, Fabrication and Analysis of Silicon Hollow Microneedles for Transdermal Drug Delivery System for Treatment of Hemodynamic Dysfunctions," Cardiovascular Engineering, vol. 10, pp. 91-108, 2010.

[4] M. W. Ashraf, S. Tayyaba, and N. Afzulpurkar, "Micro Electromechanical Systems (MEMS) Based Microfluidic Devices for Biomedical Applications," International journal of molecular sciences, vol. 12, pp. 3648-3704, 2011.

[5] M. Nafea, A. A. B. Hisham, N. A. Abdul-Kadir, and F. K. C. Harun, "Brainwave-Controlled System for Smart Home Applications," in 2018 2nd International Conference on BioSignal Analysis, Processing and Systems (ICBAPS), Sarawak, Malaysia, 2018, pp. 75-80.

[6] A. A. Badrul Hisham, M. H. I. Ishak, C. K. Teik, Z. Mohamed, and N. H. Idris, "Bluetooth-Based Home Automation System Using an Android Phone," Jurnal Teknologi (Sciences \& Engineering), vol. 70, pp. 57-61, 2014.

[7] W. Song and J. Lichtenberg, "Thermo-Pneumatic, Single-Stroke Micropump," Journal of Micromechanics and Microengineering, vol. 15, pp. 1425-1432, 2005.

[8] A. A. Badrul Hisham, S. A. Saad, M. R. R. Ahmad, R. A. Rahim, N. H. Idris, and M. H. I. Ishak, "Review of Safety Evaluation of Thermal Wearable Power Harvesting Device," International Journal of Integrated Engineering, vol. 9, pp. 76-84, 2017.

[9] M. Nafea, Z. Mohamed, A. M. Abdullahi, M. R. Ahmad, and A. R. Husain, "Dynamic Hysteresis Based Modeling of Piezoelectric Actuators," Jurnal Teknologi (Sciences and Engineering), vol. 67, pp. 9-13, 2014.

[10] M. N. Minjal, "Modeling and Control of Piezoelectric Stack Actuators with Hysteresis," Masters of Engineering (Electrical - Mechatronics \& Automatic Control), Faculty of Electrical Engineering, Universiti Teknologi Malaysia, Johor, Malaysia, 2013.

[11] A. AbuZaiter, O. F. Hikmat, M. Nafea, and M. S. Mohamed Ali, "Design and Fabrication of a Novel XY $\theta z$ Monolithic Micro-Positioning Stage Driven by Niti Shape-Memory-Alloy Actuators," Smart Materials and Structures, vol. 25, pp. 105004-105018, 2016.

Indo. J. Elec. Eng. \& Inf, Vol.7, No. 2, June 2019: 182 - 189 
[12] B. T. Chia, H.-H. Liao, and Y.-J. Yang, "A Novel Thermo-Pneumatic Peristaltic Micropump with Low Temperature Elevation on Working Fluid," Sensors and Actuators A: Physical, vol. 165, pp. 86-93, 2011.

[13] L.-J. Yang and T.-Y. Lin, "A PDMS-Based Thermo-Pneumatic Micropump with Parylene Inner Walls," Microelectronic Engineering, vol. 88, pp. 1894-1897, 2011.

[14] J.-C. Yoo, G.-S. La, C. J. Kang, and Y.-S. Kim, "Microfabricated Polydimethylsiloxane Microfluidic System Including Micropump and Microvalve for Integrated Biosensor," Current Applied Physics, vol. 8, pp. 692-695, 2008.

[15] A. Ben Amar, A. B. Kouki, and H. Cao, "Power Approaches for Implantable Medical Devices," Sensors, vol. 15, pp. 28889-28914, 2015.

[16] Y.-H. Joung, "Development of Implantable Medical Devices: From an Engineering Perspective," International Neurourology Journal, vol. 17, pp. 98-106, 2013.

[17] B. Assadsangabi, M. S. M. Ali, and K. Takahata, "Planar Variable Inductor Controlled by Ferrofluid Actuation," IEEE Transactions on Magnetics, vol. 49, pp. 1402-1406, 2013.

[18] M. S. Mohamed Ali and K. Takahata, "Wireless Microfluidic Control with Integrated Shape-Memory-Alloy Actuators Operated by Field Frequency Modulation," Journal of Micromechanics and Microengineering, vol. 21, pp. 075005-075015, 2011.

[19] P. S. Chee, M. Nafea, P. L. Leow, and M. S. Mohamed Ali, "Wireless Powered Thermo-Pneumatic Micropump Using Frequency-Controlled Heater," Sensors and Actuators A: Physical, vol. 233, pp. 1-8, 2015.

[20] P. S. Chee, M. Nafea, P. L. Leow, and M. S. Mohamed Ali, "Thermal Analysis of Wirelessly Powered ThermoPneumatic Micropump Based on Planar LC Circuit," Journal of Mechanical Science and Technology, vol. 30, pp. 2659-2665, 2016.

[21] M. Nafea, A. Nawabjan, and M. S. Mohamed Ali, "A Wirelessly-Controlled Piezoelectric Microvalve for Regulated Drug Delivery," Sensors and Actuators A: Physical, vol. 279, pp. 191-203, 2018.

[22] S. S. Mohan, M. d. M. Hershenson, S. P. Boyd, and T. H. Lee, "Simple Accurate Expressions for Planar Spiral Inductances," IEEE journal of solid-state circuits, vol. 34, pp. 1419-1424, 1999.

[23] T. Sun, X. Xie, and Z. Wang, Wireless Power Transfer for Medical Microsystems. Beijin, China: Springer, 2013.

[24] T. T. Grove, M. F. Masters, and R. E. Miers, "Determining Dielectric Constants Using a Parallel Plate Capacitor," American journal of physics, vol. 73, pp. 52-56, 2005.

[25] M. Nafea, A. AbuZiater, O. Faris, S. Kazi, and M. S. Mohamed Ali, "Selective Wireless Control of a Passive Thermopneumatic Micromixer," in 2016 IEEE 29th International Conference on Micro Electro Mechanical Systems (MEMS), Shanghai, China, 2016, pp. 792-795.

[26] S. D. Senturia, Microsystem Design. Dordrecht, Netherlands: Springer, 2001, pp. 279-297.

[27] L. Guo, G. S. Guvanasen, X. Liu, C. Tuthill, T. R. Nichols, and S. P. DeWeerth, "A PDMS-Based Integrated Stretchable Microelectrode Array (isMEA) for Neural and Muscular Surface Interfacing," IEEE transactions on biomedical circuits and systems, vol. 7, pp. 1-10, 2013.

[28] K. Lei, K.-H. Chen, and Y.-C. Chang, "Protein Binding Reaction Enhanced by Bi-Directional Flow Driven by onChip Thermopneumatic Actuator," Biomedical microdevices, vol. 16, pp. 325-332, 2014.

[29] H. K. Bardaweel and S. K. Bardaweel, "Dynamic Simulation of Thermopneumatic Micropumps for Biomedical Applications," Microsystem Technologies, vol. 19, pp. 2017-2024, 2013.

[30] Y.-J. Yang and H.-H. Liao, "Development and Characterization of Thermopneumatic Peristaltic Micropumps," Journal of Micromechanics and Microengineering, vol. 19, pp. 025003-025016, 2009.

[31] M. Nafea, A. AbuZaiter, S. Kazi, and M. S. Mohamed Ali, "Frequency-Controlled Wireless Passive Thermopneumatic Micromixer," Journal of Microelectromechanical Systems, vol. 26, pp. 691-703, 2017.

[32] V. John, "Higher Order Finite Element Methods and Multigrid Solvers in a Benchmark Problem for the 3D NavierStokes Equations," International Journal for Numerical Methods in Fluids, vol. 40, pp. 775-798, 2002.

[33] M. Mangold, J. Schmidt, L. Tobiska, and E. Tsotsas, "Modeling of Membrane Reactors," in Membrane Reactors for Hydrogen Production Processes, A. Seidel-Morgenstern, Ed. Weinheim, Germany: John Wiley \& Sons, pp. 29-62, 2010.

[34] M. Nafea, N. Ahmad, A. R. Wahap, and M. S. Mohamed Ali, "Modeling and Simulation of a Wireless Passive Thermopneumatic Micromixer," in Communications in Computer and Information Science Book Series. vol. 751, pp. 312-322, 2017.

[35] V. Mengeaud, J. Josserand, and H. H. Girault, "Mixing Processes in a Zigzag Microchannel: Finite Element Simulations and Optical Study," Analytical chemistry, vol. 74, pp. 4279-4286, 2002. 